\title{
Author Correction: High-speed volumetric imaging of neuronal activity in freely moving rodents
}

Oliver Skocek, Tobias Nöbauer D, Lukas Weilguny D, Francisca Martínez Traub, Chuying Naomi Xia, Maxim I. Molodtsov, Abhinav Grama, Masahito Yamagata (D), Daniel Aharoni, David D. Cox, Peyman Golshani and Alipasha Vaziri (D)

Correction to: Nature Methods https://doi.org/10.1038/s41592-018-0008-0, published online 7 May 2018.

In the version of this Brief Communication originally published online, ref. 21 included details for a conference paper (Pegard, N. C. et al. Paper presented at Novel Techniques in Microscopy: Optics in the Life Sciences, Vancouver, BC, Canada, 12-15 April 2015). The correct reference is the following: Pégard, N. C. et al. Optica 3, 517-524 (2016). This error has been corrected in the print, HTML and PDF versions of the paper. 